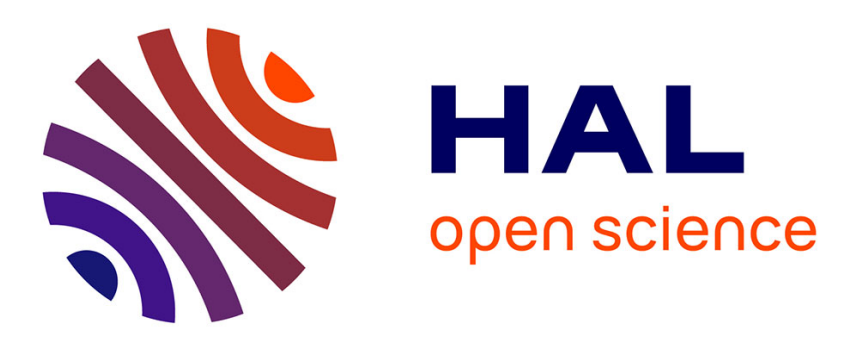

\title{
VaR and ES for linear portfolios with mixture of elliptic distributions Risk Factors
}

\author{
Jules Sadefo-Kamdem
}

\section{To cite this version:}

Jules Sadefo-Kamdem. VaR and ES for linear portfolios with mixture of elliptic distributions Risk Factors. 2003. hal-00001214

\section{HAL Id: hal-00001214 \\ https://hal.science/hal-00001214}

Preprint submitted on 27 Feb 2004

HAL is a multi-disciplinary open access archive for the deposit and dissemination of scientific research documents, whether they are published or not. The documents may come from teaching and research institutions in France or abroad, or from public or private research centers.
L'archive ouverte pluridisciplinaire HAL, est destinée au dépôt et à la diffusion de documents scientifiques de niveau recherche, publiés ou non, émanant des établissements d'enseignement et de recherche français ou étrangers, des laboratoires publics ou privés. 


\title{
VAR AND ES FOR LINEAR PORTFOLIOS WITH MIXTURE OF ELLIPTIC DISTRIBUTED RISK FACTORS
}

\author{
JULES SADEFO KAMDEM \\ UNIVERSITÉ DE REIMS \\ UNIVERSITÉ D'EVRY
}

\begin{abstract}
In this paper, following the generalization of Delta Normal VaR to Delta Mixture Elliptic VaR in Sadefo-Kamdem [3], we give and explicit formula to estimate linear $\mathrm{VaR}$ and ES when the risk factors changes with the mixture of $t$-Student distributions. In particular, we give rise to Delta-Mixture-Student VaR and the Delta-Mixture-Elliptic ES.
\end{abstract}

Key Words: Mixture of Elliptic distributions, Linear portfolio, Value-at-Risk, Expected Shortfall, Capital allocation.

\section{Introduction}

The original RiskMetrics methodology for estimating VaR was based on parametric methods, and used the multi-variate normal distribution. This approach works well for the so-called linear portfolios, that is, those portfolios whose aggregate return is, to a good approximation, a linear function of the returns of the individual assets which make up the portfolio, and in situations where the latter can be assumed to be jointly normally distributed. For other portfolios, like portfolios of derivatives depending non-linearly on the return of the underlying, or portfolios of non-normally distributed assets, one generally turns to Monte Carlo methods to estimate the VaR. This is an issue in situations demanding for real-time evaluation of financial risk. For non-linear portfolios, practitioners, as an alternative to Monte Carlo, use $\Delta$-normal VaR methodology, in which the portfolio return is linearly approximated, and an assumption of normality is made. Such methods present us with a trade-off between accuracy and speed, in the sense that they are much faster than Monte Carlo, but are much less accurate unless the linear approximation is quite good and the normality hypothesis holds well. The assumption of normality simplifies the computation of VaR considerably. However it is inconsistent with the empirical evidence of assets returns, which finds that asset returns are fat tailed. This implies that extreme events are much more likely to occur in practice than would be predicted based on the assumption of normality.

Some alternative return distributions have been proposed in the world of elliptic distributions by Sadefo-Kamdem [3], that better reflect the empirical evidence. In this paper, following [3], I examine one such alternative that simultaneously allows for asset returns that are fat tailed and for tractable calculation of Value-at-Risk and Expected Shortfall, by giving attention to mixture of elliptic distributions,

This draft is a part of J.SADEFO-KAMDEM PhD Thesis at the Université de Reims (France). The author is a temporary lecturer at the Department of Mathematics ( Université d'evry Val d'essonne France). Author's address: Université de Reims, Laboratoire de Mathématiques UMR 6056-CNRS, BP 1039 Moulin de la Housse, 51687 Reims cedex 2 FRANCE. e-mail: sadefo@univ-reims.fr. 
with an explicit formula of VaR and ES in the special case where assets returns changes with mixture of Student- $t$ distributions. Note that, the particular case based on mixture of normal distributions, has been proposed by Zangari(1996)河, Subu-Venkataraman [5] and some references therein.

An obvious first generalization is to keep the linearity assumption, but replace the normal distribution by some other family of multi-variate distributions. In SadefoKamdem [3], we have such generalization concerning linear portfolios, in the case where the joint risk factors changes with mixture of elliptic distributions. In this paper, by using some generalized theorems concerning Delta-Mixture-Elliptic VaR and Delta-Student VaR in [3], we introduce the notion of Delta-Mixture-Student VaR, Delta-Mixture-Elliptic ES and the Delta-Mixture-Student ES.

So the particular subject of this paper, is to give an explicit formulas that will permit to obtain the linear VaR or linear ES, when the joint risk factors of the linear portfolios, changes with mixture of $t$-Student distributions. Note that, since one shortcoming of the multivariate t-distribution is that all the marginal distributions must have the same degrees of freedom, which implies that all risk factors have equally heavy tails, the mixture of $t$-Student will be view as a serious alternatives, to a simple $t$-Student-distribution. Therefore, the methodology proposes by this paper seem to be interesting to controlled thicker tails than the standard Student distribution.

The paper is organized, as follows: In section 2, we recall some theorems concerning the Delta-Elliptic, Delta-Elliptic and Delta Mixture Elliptic VaR given by Sadefo-Kamdem [3]. In section 3, following the theorem concerning Delta Mixture Elliptic VaR, we show how to reduce the computation of the Delta-Mixture-Student $V a R$ to finding the zeros of a mixture of special function. In section 4 , we introduces the notion of Delta Mixture Elliptic ES, by treat the expected shortfall for general mixture of elliptic distribution, with special attention to Delta Mixture Elliptic ES . Finally, in section 5 we discuss some potential application areas.

\section{Some Notions on LOG-Elliptic Linear VaR}

In this section, following [3], we recall some notions on elliptic distributions and Linear VaR.

We will use the following notational conventions for the action of matrices on vectors: single letters $x, y, \cdots$ will denote row vectors $\left(x_{1}, \cdots, x_{n}\right),\left(y_{1}, \cdots y_{n}\right)$. The corresponding column vectors will be denoted by $x^{t}, y^{t}$, the ${ }^{t}$ standing more generally for taking the transpose of any matrix. Matrices $A=\left(A_{i j}\right)_{i, j}, B$, etc. will be multiplied in the usual way. In particular, $A$ will act on vectors by leftmultiplication on column vectors, $A y^{t}$, and by right multiplication on row vectors, $x A ; x \cdot x=x x^{t}=x_{1}^{2}+\cdots+x_{n}^{2}$ will stand for the Euclidean inner product.

A portfolio with time- $t$ value $\Pi(t)$ is called linear if its profit and loss $\Delta \Pi(t)=$ $\Pi(t)-\Pi(0)$ over a time window, $[0 \mathrm{t}]$ is a linear function of the returns $X_{1}(t), \ldots, X_{n}(t)$ of its constituents over the same time period:

$$
\Delta \Pi(t)=\delta_{1} X_{1}+\delta_{2} X_{2}+\ldots+\delta_{n} X_{n}
$$

This will for instance be the case for ordinary portfolios of common stock, if we use percentage returns, and will also hold to good approximation with log-returns, provided the time window $[0, \mathrm{t}]$ is small. We will drop the time $t$ from our notations, since it will be kept fixed, and simply write $X_{j}, \Delta \Pi$, etc. We also put

$$
X=\left(X_{1}, \cdots, X_{n}\right)
$$

so that $\Delta \Pi=\delta \cdot X=\delta X^{t}$. 
We now assume that the $X_{j}$ are elliptically distributed with mean $\mu$ and correlation matrix $\Sigma=A A^{t}$ :

$$
\left(X_{1}, \ldots, X_{n}\right) \sim N(\mu, \Sigma, \phi) .
$$

This means that the pdf of $\mathrm{X}$ is of the form

$$
f_{X}(x)=|\Sigma|^{-1 / 2} g\left((x-\mu) \Sigma^{-1}(x-\mu)^{t}\right),
$$

where $|\Sigma|$ stands for the determinant of $\Sigma$, and where $g: \mathbb{R}_{\geq 0} \rightarrow 0$ is such that the Fourier transform of $g\left(|x|^{2}\right)$, as a generalized function on $\mathbb{R}^{n}$, is equal to $\phi\left(|\xi|^{2}\right)$

1.

Assuming that $g$ is continuous, and non-zero everywhere, the Value at Risk at a confidence level of $1-\alpha$ is given by solution of the following equation:

Here we follow the usual convention of recording portfolio losses by negative numbers, but stating the Value-at-Risk as a positive quantity of money.

2.1. Linear VaR with mixtures of elliptic Distributions. Mixture distributions can be used to model situations where the data can be viewed as arising from two or more distinct classes of populations; see also [1]. For example, in the context of Risk Management, if we divide trading days into two sets, quiet days and hectic days, a mixture model will be based on the fact that returns are moderate on quiet days, but can be unusually large or small on hectic days. Practical applications of mixture models to compute VaR can be found in Zangari [7] (1996), who uses a mixture normal to incorporate fat tails in VaR estimation. In SadefoKamdem [3], we have generalized the preceding section to the situation where the joint log-returns follow a mixture of elliptic distributions, that is, a convex linear combination of elliptic distributions. In this section, a special attention will be give to mixture of Student- $t$ distributions.

Definition 2.1. We say that $\left(X_{1}, \ldots, X_{n}\right)$ has a joint distribution that is the mixture of $m$ elliptic distributions $N\left(\mu_{j}, \Sigma_{j}, \phi_{j}\right)^{2}$, with weights $\left\{\beta_{j}\right\} \quad\left(\mathrm{j}=1, . ., \mathrm{m} ; \beta_{j}>0\right.$ $; \sum_{j=1}^{m} \beta_{j}=1$ ), if its cumulative distribution function can be written as

$$
F_{X_{1}, \ldots, X_{n}}\left(x_{1}, \ldots, x_{n}\right)=\sum_{j=1}^{m} \beta_{j} F_{j}\left(x_{1}, \ldots, x_{n}\right)
$$

with $F_{j}\left(x_{1}, \ldots, x_{n}\right)$ the cdf of $N\left(\mu_{j}, \Sigma_{j}, \phi_{j}\right)$.

Remark 2.2. In practice, one would usually limit oneself to $m=2$, due to estimation and identification problems; see [1].

The following lemma is given by Sadefo-Kamdem [3].

Lemma 2.3. Let $\Delta \Pi=\delta_{1} X_{1}+\ldots+\delta_{n} X_{n}$ with $\left(X_{1}, \ldots, X_{n}\right)$ is a mixture of elliptic distributions, with density

$$
f(x)=\sum_{j=1}^{m} \beta_{j}\left|\Sigma_{j}\right|^{-1 / 2} g_{j}\left(\left(x-\mu_{j}\right) \Sigma_{j}^{-1}\left(x-\mu_{j}\right)^{t}\right)
$$

where $\mu_{j}$ is the vector mean, and $\Sigma_{j}$ the variance-covariance matrix of the $j$-th component of the mixture. We suppose that each $g_{j}$ is integrable function over $\mathbb{R}$, and that the $g_{j}$ never vanish jointly in a point of $\mathbb{R}^{m}$. Then the value-at-Risk,

\footnotetext{
${ }^{1}$ One uses $\phi$ as a parameter for the class of elliptic distributions, since it is always well-defined as a continuous function: $\phi\left(|\xi|^{2}\right)$ is simply the characteristic function of an $X \sim N(0, I d, \phi)$. Note, however, that in applications we'd rather know $g$

$2_{\text {or }} N\left(\mu_{j}, \Sigma_{j}, g_{j}\right)$ if we parameterize elliptical distributions using $g$ instead of $\phi$
} 
or Delta mixture-elliptic VaR, at confidence $1-\alpha$ is given as the solution of the transcendental equation

$$
\alpha=\sum_{j=1}^{m} \beta_{j} G_{j}\left(\frac{\delta \cdot \mu_{j}^{t}+V a R_{\alpha}}{\left(\delta \Sigma_{j} \delta\right)^{1 / 2}}\right),
$$

where

$$
G_{j}=\frac{\left|S_{n-2}\right|}{\left|\Sigma_{j}\right|^{1 / 2}} \int_{0}^{+\infty} r^{n-2}\left[\int_{-\infty}^{\frac{-\delta \cdot \mu_{j}-V a R_{\alpha}}{\left|\delta A_{j}\right|}} g_{j}\left(z_{1}^{2}+r^{2}\right) d z_{1}\right] d r .
$$

Here $\delta=\left(\delta_{1}, \ldots, \delta_{n}\right)$.

Remark 2.4. In the case of a mixture of $m$ elliptic distributions the VaR will not depend any more in a simple way on the total portfolio mean and variancecovariance. This is unfortunate, but already the case for a mixture of normal distributions.

Remark 2.5. One might, in certain situations, try to model with a mixture of elliptic distributions which all have the same variance-covariance and the same mean, and obtain for example a mixture of different tail behaviors by playing with the $g_{j}$ 's. In that case the VaR again simplifies to: $V a R_{\alpha}=-\delta \cdot \mu+q_{\alpha} \cdot \sqrt{\delta \Sigma \delta^{t}}$, with $q_{\alpha}$ now the unique positive solution to

$$
\alpha=\sum_{j=1}^{m} \beta_{j} G_{j}\left(q_{\alpha}\right) .
$$

The preceding can immediately be specialized to a mixture of normal distributions. the details is left to the reader.

\section{VAR with mixture Student- $t$ Distributions}

We now consider in detail the case where our mixture of elliptic distributions is a mixture of multivariate Student- $t$. We will, unsurprisingly, call the corresponding VaR the Delta mixture-Student VaR.

In the case of our mixture of multi-variate t-Student distributions, the portfolio probability density function is given by:

$$
h_{X}(x)=\sum_{j=1}^{m} \beta_{j} \frac{\Gamma\left(\frac{\nu_{j}+n}{2}\right)}{\Gamma\left(\nu_{j} / 2\right) \cdot \sqrt{\left|\Sigma_{j}\right|\left(\nu_{j} \pi\right)^{n}}}\left(1+\frac{\left(x-\mu_{j}\right)^{t} \Sigma_{j}^{-1}\left(x-\mu_{j}\right)}{\nu_{j}}\right)^{\left(\frac{-\nu_{j}-n}{2}\right)},
$$

$x \in \mathbb{R}^{n}$ and $\nu_{j}>2$. Hence $g_{j}$ is given by

$$
g_{j}(s)=C\left(\nu_{j}, n\right)\left(1+s / \nu_{j}\right)^{-\frac{\left(n+\nu_{j}\right)}{2}}, \quad s \geq 0,
$$

where we have put

$$
C\left(\nu_{j}, n\right)=\frac{\Gamma\left(\frac{\nu_{j}+n}{2}\right)}{\Gamma\left(\nu_{j} / 2\right) \sqrt{\left(\nu_{j} \pi\right)^{n}}} .
$$

Using this $g_{j}$ in (1), we find that

$$
G_{j}(s)=\frac{\nu_{j}^{\frac{n+\nu_{j}}{2}}}{2}\left|S_{n-2}\right| C\left(\nu_{j}, n\right) \int_{s}^{\infty} I_{j}\left(z_{1}\right) d z_{1},
$$

where we have put

$$
I_{j}\left(z_{1}\right)=\int_{z_{1}^{2}}^{+\infty}\left(u-z_{1}^{2}\right)^{\frac{n-3}{2}}\left(\nu_{j}+u\right)^{-\frac{\left(n+\nu_{j}\right)}{2}} d u .
$$


Following [3], we have the following expression

$$
G_{j}(s)=\frac{1}{\nu_{j} \sqrt{\pi}}\left(\frac{\nu_{j}}{s^{2}}\right)^{\nu_{j} / 2} \frac{\Gamma\left(\frac{\nu_{j}+1}{2}\right)}{\Gamma\left(\frac{\nu_{j}}{2}\right)}{ }_{2} F_{1}\left(\frac{1+\nu_{j}}{2}, \frac{\nu_{j}}{2} ; 1+\frac{\nu_{j}}{2} ;-\frac{\nu_{j}}{s^{2}}\right)
$$

we obtain the following corollary

Corollary 3.1. Let $\Delta \Pi=\delta_{1} X_{1}+\ldots+\delta_{n} X_{n}$ with $\left(X_{1}, \ldots, X_{n}\right)$ is a mixture of $m$ $t$-Student distributions, with density $h_{X}$ defined by (2), where $\mu_{j}$ is the vector mean, and $\Sigma_{j}$ the variance-covariance matrix of the $j$-th component of the mixture. Then the value-at-Risk, or Delta mixture-student VaR, at confidence $1-\alpha$ is given as the solution of the transcendental equation

(6)

$\alpha=\sum_{j=1}^{m} \frac{\beta_{j} \Gamma\left(\frac{\nu_{j}+1}{2}\right)}{\nu_{j} \sqrt{\pi} \Gamma\left(\frac{\nu_{j}}{2}\right)} \nu_{j}^{\frac{\nu_{j}}{2}}\left(\frac{\delta . \mu_{j}^{t}+V a R_{\alpha}}{\delta \Sigma_{j} \delta}\right)^{\frac{-\nu_{j}}{2}}{ }_{2} F_{1}\left(\frac{1+\nu_{j}}{2}, \frac{\nu_{j}}{2} ; 1+\frac{\nu_{j}}{2} ;-\frac{\nu_{j}\left(\delta . \mu_{j}^{t}+V a R_{\alpha}\right)}{\delta \Sigma_{j} \delta}\right)$

where $G_{j}$ is defined by (马) with $g=g_{j}$. Here $\delta=\left(\delta_{1}, \ldots, \delta_{n}\right)$.

Corollary 3.2. One might, in certain situations, try to model with a mixture of $t$-Student distributions which all have the same variance-covariance $\Sigma=\Sigma_{j}$ and the same mean $\mu=\mu_{j}$, and obtain for example a mixture of different tail behaviors by playing with the $\nu_{j}$ 's. In that case the VaR again simplifies to:

$$
V a R_{\alpha}=-\delta \cdot \mu+q_{\alpha} \cdot \sqrt{\delta \Sigma \delta^{t}}
$$

with $q_{\alpha}$ now the unique positive solution to

$\alpha=\sum_{j=1}^{m} \frac{\beta_{j} \Gamma\left(\frac{\nu_{j}+1}{2}\right)}{\nu_{j} \sqrt{\pi} \Gamma\left(\frac{\nu_{j}}{2}\right)} \nu_{j}{ }^{\frac{\nu_{j}}{2}}\left(\frac{\delta . \mu^{t}+V a R_{\alpha}}{\delta \Sigma \delta}\right)^{\frac{-\nu_{j}}{2}}{ }_{2} F_{1}\left(\frac{1+\nu_{j}}{2}, \frac{\nu_{j}}{2} ; 1+\frac{\nu_{j}}{2} ;-\frac{\nu_{j}\left(\delta . \mu^{t}+V a R_{\alpha}\right)}{\delta \Sigma \delta}\right)$.

Remark 3.3. One might, in certain situations, try to model with a mixture of $t$-Student distributions which all have the same $\nu_{j}=\nu$ and the same mean $\mu_{j} \approx 0$, and obtain for example a mixture of different tail behaviors by playing with the $\Sigma_{j}$ 's. In that case the $\mathrm{VaR}$ is the unique positive solution to

$$
\alpha=\frac{\Gamma\left(\frac{\nu+1}{2}\right)}{\nu \sqrt{\pi} \Gamma\left(\frac{\nu}{2}\right)} \sum_{j=1}^{m} \beta_{j}\left(\frac{\nu\left(V a R_{\alpha}\right)}{\delta \Sigma_{j} \delta}\right)_{2}^{\frac{\nu}{2}} F_{1}\left(\frac{1+\nu}{2}, \frac{\nu}{2} ; 1+\frac{\nu}{2} ;-\frac{\nu\left(V a R_{\alpha}\right)}{\delta \Sigma_{j} \delta}\right) .
$$

3.1. Some Numerical Result of Delta Mixture-Student VaR coefficient. Here we give some numerical results when applying the corollary 3.2, in the situation where $m=2$.

By introducing the function $F$ such that

$$
F\left(s, \beta, \nu_{1}, \nu_{2}\right)=\beta \cdot G_{1}(s)+(1-\beta) \cdot G_{2}(s),
$$

where $G_{j}$ is define in (5), for $j=1,2$, for given as inputs $\beta, \nu_{1}$ and $\nu_{2}$, we give a table that contains some solutions $s=q_{\beta, \nu_{1}, \nu_{2}}=q_{\alpha}^{M S-V a R}$ of the following transcendental equation:

$$
F\left(s, \beta, \nu_{1}, \nu_{2}\right)=\alpha .
$$

For given $\Sigma, \mu$, and $\delta$, these solutions will permit to calculate $V a R_{\alpha}$, when the confidence is $1-\alpha$. 
(1) In the case where $\alpha=0.01$, we obtain some solutions of (3.1) in the following table:

\begin{tabular}{|c|c|c|c|c|c|c|c|c|c|c|c|}
\hline$\left(\nu_{1}, \nu_{2}\right)$ & $(2,3)$ & $(3,4)$ & $(4,6)$ & $(5,8)$ & $(6,10)$ & \multicolumn{2}{|c|}{$(7,15)$} & \multicolumn{2}{|c|}{$(8,40)$} & \multicolumn{2}{|c|}{$(9,16)$} \\
\hline$q_{0.05, \nu_{1}, \nu_{2}}$ & 4.64839 & 3.78507 & 3.17184 & .91919 & 2.78228 & \multicolumn{2}{|c|}{2.62175} & \multicolumn{2}{|c|}{2.44602} & \multicolumn{2}{|c|}{2.59524} \\
\hline & 4.7586 & 3.82348 & 3.20124 & 2.94213 & 2.80092 & \multicolumn{2}{|c|}{2.64116} & \multicolumn{2}{|c|}{2.46906} & \multicolumn{2}{|c|}{2.60704} \\
\hline$q_{0.15}$ & 4.87115 & 3.86216 & 3.23086 & 2.9652 & 2.81965 & \multicolumn{2}{|c|}{2.6607} & \multicolumn{2}{|c|}{2.49235} & \multicolumn{2}{|c|}{2.61887} \\
\hline$\overline{q_{0.20, \nu_{1}, \nu_{2}}}$ & 4.98587 & 3.9011 & 3.26066 & 2.98846 & 2.83846 & \multicolumn{2}{|c|}{2.68035} & \multicolumn{2}{|c|}{2.51586} & \multicolumn{2}{|c|}{2.63073} \\
\hline$q_{0.25, \nu_{1}, \nu_{2}}$ & 5.10258 & 3.94025 & 3.29063 & 3.01177 & \multirow{2}{*}{2.85734} & \multicolumn{2}{|c|}{2.70009} & \multicolumn{2}{|c|}{2.53957} & \multicolumn{2}{|c|}{2.64261} \\
\hline$\overline{q_{0.30, \nu_{1}, \nu_{2}}}$ & 5.22106 & 3.97962 & 3.32075 & 3.03518 & & \multicolumn{2}{|c|}{2.71991} & \multicolumn{2}{|c|}{2.56344} & \multicolumn{2}{|c|}{2.65452} \\
\hline$q_{0.35, \nu_{1}, \nu_{2}}$ & 5.34113 & 4.01917 & 3.35100 & 3.05866 & $\frac{2.87629}{2}$ & \multirow{2}{*}{\multicolumn{2}{|c|}{$\frac{2.7398}{2.75974}$}} & \multicolumn{2}{|c|}{2.58744} & \multicolumn{2}{|c|}{2.66644} \\
\hline$q_{0.40, \nu_{1}, \nu_{2}}$ & 5.46259 & 4.05888 & 3.38136 & 3.08221 & 2.91432 & & & 2.6 & & 2.67 & \\
\hline$q_{0.45, \nu_{1}, \nu_{2}}$ & 5.58523 & 4.09873 & 3.41180 & 3.10502 & 2.93339 & & 7972 & 2.6 & & 2.69 & \\
\hline$q_{0.50, \nu_{1}, \nu_{2}}$ & 5.70886 & 4.13870 & 3.44231 & 3.12946 & 2.95248 & & 9972 & 2.6 & 989 & 2.70 & \\
\hline$\overline{\left(\nu_{1}, \nu_{2}\right)}$ & $\overline{(1(10,20)}$ & (20,30) & $\overline{(200,300)}$ & $\overline{(250,}$ & $\overline{(275}$ & & $\overline{(300}$ & & $\overline{(40}$ & $\overline{0)}$ & $\overline{\overline{(1}}$ \\
\hline$q_{0.05, \nu_{1}, \nu_{2}}$ & 2.53963 & 2.46079 & 2.33916 & 2.4001 & 2.58 & & 2.39 & & & & 3.3202 \\
\hline$\overline{q_{0.10, \nu_{1}, \nu_{2}}}$ & 2.55132 & 2.46432 & 2.33947 & 2.3970 & 2.576 & & 2.39 & & 2.7 & & 3.27401 \\
\hline$\overline{q_{0.15, \nu_{1}, \nu_{2}}}$ & 2.56304 & 2.46785 & 2.33978 & 2.3939 & 2.563 & & 2.38 & & 2.7 & & 3.22632 \\
\hline$q_{0.20, \nu_{1}, \nu_{2}}$ & 2.5748 & 2.47139 & 2.3401 & 2.390 & 2.550 & & 2.38 & & 2.68 & 019 & 3.17715 \\
\hline$q_{0.25, \nu_{1}, \nu_{2}}$ & 2.58658 & 2.47492 & 2.34041 & 2.387 & 2.537 & & 2.38 & & 2.6 & & 3.12651 \\
\hline$\overline{q_{0.30, \nu_{1}, \nu_{2}}}$ & 2.59838 & 2.47846 & 2.34073 & 2.3847 & 2.524 & & 2.37 & & 2.63 & & 3.07446 \\
\hline$q_{0.35, \nu_{1}, \nu_{2}}$ & 2.6102 & 2.482 & 2.34104 & 2.3816 & 2.5 & & 2.37 & & 2.61 & & 3.02112 \\
\hline$q_{0.40, \nu_{1}, \nu_{2}}$ & 2.62204 & 2.48553 & 2.34136 & 2.3785 & 2.497 & & 2.37 & & $2.5 \mathrm{~s}$ & & 2.96663 \\
\hline$q_{0.45, \nu_{1}, \nu_{2}}$ & 2.63389 & 2.48907 & 2.34167 & 2.3754 & 2.484 & & 2.37 & 33 & 2.57 & & 2.91121 \\
\hline$q_{0.50,}$ & 2.64574 & 2.49261 & 2.34199 & 2.372 & 2.4 & & 2.3 & 46 & 2.5 & 009 & 2.85513 \\
\hline
\end{tabular}

(2) In the case where $\alpha=0.001$, we obtain some solutions of (3.1) in the following table:

\begin{tabular}{|c|c|c|c|c|c|c|c|c|}
\hline$\left(\nu_{1}, \nu_{2}\right)$ & $(2,3)$ & $(3,4)$ & $(4,6)$ & $(5,8)$ & $(6,10)$ & $(7,15)$ & $(8,40)$ & $(9,16)$ \\
\hline$q_{0.20, \nu_{1}, \nu_{2}}$ & 12.8878 & 7.84891 & 5.66393 & 4.82769 & 4.39245 & 3.98902 & 3.62286 & 3.82625 \\
\hline$q_{0.25, \nu_{1}, \nu_{2}}$ & 13.5577 & 8.01412 & 5.77451 & 4.90665 & 4.45334 & 4.05064 & 3.69896 & 3.86013 \\
\hline$q_{0.30, \nu_{1}, \nu_{2}}$ & 14.2205 & 8.17734 & 5.88317 & 4.98414 & 4.51241 & 4.11084 & 3.77242 & 3.89346 \\
\hline$q_{0.35, \nu_{1}, \nu_{2}}$ & 14.874 & 8.33840 & 5.98975 & 5.06004 & 4.57030 & 4.16948 & 3.84285 & 3.92621 \\
\hline$q_{0.40, \nu_{1}, \nu_{2}}$ & 15.5168 & 8.49717 & 6.09412 & 5.13427 & 4.62694 & 4.22648 & 3.91007 & 3.95838 \\
\hline$q_{0.45, \nu_{1}, \nu_{2}}$ & 16.1480 & 8.65357 & 6.19624 & 5.20677 & 4.68229 & 4.28179 & 3.97400 & 3.98993 \\
\hline$q_{0.50, \nu_{1}, \nu_{2}}$ & 16.7671 & 8.80753 & 6.29604 & 5.27752 & 4.73634 & 4.33537 & 3.03470 & 4.02087 \\
\hline
\end{tabular}

(3) In the case where $\alpha=0$, we obtain some solutions of (3.1) in the following table:

\begin{tabular}{|c|c|c|c|c|c|c|c|c|}
\hline$\left(\nu_{1}, \nu_{2}\right)$ & $(2,3)$ & $(3,4)$ & $(4,6)$ & $(5,8)$ & $(6,10)$ & $(7,15)$ & $(8,40)$ & $(9,16)$ \\
\hline$q_{0.20, \nu_{1}, \nu_{2}}$ & 322.785 & 82.6688 & 31.0894 & 20.7154 & 15.8813 & 11.4371 & 10.1089 & 9.25604 \\
\hline$q_{0.25, \nu_{1}, \nu_{2}}$ & 352.09 & 87.1881 & 32.5561 & 21.541 & 16.42471 & 11.7949 & 10.3957 & 9.47529 \\
\hline$q_{0.30, \nu_{1}, \nu_{2}}$ & 378.302 & 91.2285 & 33.8309 & 22.2487 & 16.88721 & 12.0958 & 10.6352 & 9.66243 \\
\hline$q_{0.35, \nu_{1}, \nu_{2}}$ & 402.155 & 94.8927 & 34.9619 & 22.8697 & 17.2907 & 12.3561 & 10.8414 & 9.82571 \\
\hline$q_{0.40, \nu_{1}, \nu_{2}}$ & 424.137 & 98.2529 & 35.981 & 23.4244 & 17.6493 & 12.5858 & 11.0227 & 9.97061 \\
\hline$q_{0.45, \nu_{1}, \nu_{2}}$ & 444.591 & 101.362 & 36.9102 & 23.9265 & 17.9726 & 12.7919 & 11.1848 & 10.1009 \\
\hline$q_{0.50, \nu_{1}, \nu_{2}}$ & 463.771 & 104.26 & 37.7655 & 24.3858 & 18.2673 & 12.9789 & 11.3316 & 10.2194 \\
\hline
\end{tabular}

Remark 3.4. Note that, the precedent results are available when $\alpha=0$. This means that with our model, one would calculate the linear VaR with mixture of elliptic distributions, for 100 percent confidence level. 


\section{Expected Shortfall With mixture of Elliptic Distributions}

Expected shortfall is a sub-additive risk statistic that describes how large losses are on average when they exceed the VaR level. Expected shortfall will therefore give an indication of the size of extreme losses when the VaR threshold is breached. We will evaluate the expected shortfall for a linear portfolio under the hypothesis of mixture of elliptically distributed risk factors. Mathematically, the expected shortfall associated with a given VaR is defined as:

$$
\text { Expected Shortfall }=\mathbb{E}(-\Delta \Pi \mid-\Delta \Pi>\operatorname{VaR}),
$$

see for example [1]. Assuming again a multivariate mixture of elliptic probability density $f_{X}(x)=\sum_{i=1}^{n} \beta_{i}\left|\Sigma_{i}\right|^{-1 / 2} g_{i}\left(\left(x-\mu_{i}\right) \Sigma_{i}^{-1}\left(x-\mu_{i}\right)^{t}\right)$, the Expected Shortfall at confidence level $1-\alpha$ is given by

$$
\begin{aligned}
-E S_{\alpha} & =\mathbb{E}\left(\Delta \Pi \mid \Delta \Pi \leq-V a R_{\alpha}\right) \\
& =\frac{1}{\alpha} \mathbb{E}\left(\Delta \Pi \cdot 1_{\left\{\Delta \Pi \leq-V a R_{\alpha}\right\}}\right) \\
& =\frac{1}{\alpha} \int_{\left\{\delta x^{t} \leq-V a R_{\alpha}\right\}} \delta x^{t} f_{X}(x) d x \\
& =\sum_{i=1}^{n} \beta_{i} \frac{\left|\Sigma_{i}\right|^{-1 / 2}}{\alpha} \int_{\left\{\delta x^{t} \leq-V a R_{\alpha}\right\}} \delta x^{t} g_{i}\left(\left(x-\mu_{i}\right) \Sigma_{i}^{-1}\left(x-\mu_{i}\right)^{t}\right) d x
\end{aligned}
$$

Let $\Sigma=A_{i}^{t} A_{i}$, as before.Doing the same linear changes of variables as in section 2, we arrive at:

$$
\begin{aligned}
-E S_{\alpha} & =\frac{1}{\alpha} \sum_{i=1}^{m} \beta_{i} \int_{\left\{\left|\delta A_{i}\right| z_{1} \leq-\delta \cdot \mu_{i}-V a R_{\alpha}\right\}}\left(|\delta A| z_{1}+\delta \cdot \mu_{i}\right) g_{i}\left(\|z\|^{2}\right) d z \\
& =\frac{1}{\alpha} \sum_{i=1}^{m} \beta_{i}\left[\int_{\left\{|\delta A| z_{1} \leq-\delta \cdot \mu_{i}-V a R_{\alpha}\right\}}\left|\delta A_{i}\right| z_{1} g_{i}\left(\|z\|^{2}\right) d z+\delta \cdot \mu_{i}\right] .
\end{aligned}
$$

The final integral on the right hand side can be treated as before, by writing $\|z\|^{2}=$ $z_{1}^{2}+\left\|z^{\prime}\right\|^{2}$ and introducing spherical coordinates $z^{\prime}=r \xi, \xi \in S_{n-2}$, leading to:

$-E S_{\alpha}=\sum_{i=1}^{m} \beta_{i} \delta \cdot \mu_{i}+\frac{\left|S_{n-2}\right|}{\alpha} \sum_{i=1}^{m} \beta_{i} \int_{0}^{\infty} r^{n-2}\left[\int_{-\infty}^{\frac{-\delta \mu_{i}^{t}-V a R_{\alpha}}{\left|\delta A_{i}\right|}}\left|\delta A_{i}\right| z_{1} g_{i}\left(z_{1}^{2}+r^{2}\right) d z_{1}\right] d r$

We now first change $z_{1}$ into $-z_{1}$, and then introduce $u=z_{1}^{2}+r^{2}$, as before. If we recall that, by theorem 2.1 ,

$$
q_{\alpha, i}^{g}=\frac{\delta \cdot \mu_{i}+V a R_{\alpha}}{\left|\delta A_{i}\right|}
$$

then, simply writing $q_{\alpha, i}$ for $q_{\alpha, n}^{f_{X}}$, we arrive at:

$$
\begin{aligned}
E S_{\alpha} & =-\sum_{i=1}^{m} \beta_{i}\left[\delta \cdot \mu_{i}+|\delta A| \frac{\left|S_{n-2}\right|}{\alpha} \cdot \int_{q_{\alpha, i}}^{\infty} \int_{z_{1}^{2}}^{\infty} z_{1}\left(u-z_{1}^{2}\right)^{\frac{n-3}{2}} g(u) d u d z_{1}\right] \\
& =-\sum_{i=1}^{m} \beta_{i}(\delta \cdot \mu)+\sum_{i=1}^{n} \beta_{i}\left|\delta A_{i}\right| \frac{\left|S_{n-2}\right|}{\alpha} \cdot \int_{q_{\alpha, i}^{2}}^{\infty} \frac{1}{n-1}\left(u-q_{\alpha, i}^{2}\right)^{\frac{n-1}{2}} g_{i}(u) d u
\end{aligned}
$$

since

$$
\int_{q_{\alpha, i}}^{\sqrt{u}} z_{1}\left(u-z_{1}^{2}\right)^{\frac{n-3}{2}} d z_{1}=\frac{1}{n-1}\left(u-q_{\alpha, i}^{2}\right)^{\frac{n-1}{2}} .
$$

After substituting the formula for $\left|S_{n-2}\right|$ and using the functional equation for the $\Gamma$-function, $\Gamma(x+1)=x \Gamma(x)$, we arrive at the following result: 
Theorem 4.1. Suppose that the portfolio is linear in the risk-factors $X=\left(X_{1}, \cdots, X_{n}\right)$ : $\Delta \Pi=\delta \cdot X$ and that $X \sim N(\mu, \Sigma, \phi)$, with pdf $f_{X}(x)=\sum_{i=1}^{n} \beta_{i}\left|\Sigma_{i}\right|^{-1 / 2} g((x-$ $\left.\left.\mu_{i}\right) \Sigma_{i}^{-1}\left(x-\mu_{i}\right)^{t}\right)$. If we replace $q_{\alpha}$ by his value, then the expected Shortfall at level $\alpha$ is given by :

$$
E S_{\alpha}=-\sum_{i=1}^{m} \beta_{i}\left(\delta \cdot \mu_{i}\right)+\sum_{i=1}^{m} \beta_{i}\left|\delta \Sigma_{i} \delta^{t}\right|^{1 / 2} \cdot \frac{\pi^{\frac{n-1}{2}}}{\alpha \cdot \Gamma\left(\frac{n+1}{2}\right)} \cdot \int_{\left(q_{\alpha, i}^{g}\right)^{2}}^{\infty}\left(u-\left(q_{\alpha, i}^{g}\right)^{2}\right)^{\frac{n-1}{2}} g_{i}(u) d u \text {. }
$$

Remark 4.2. If we are in situations where $\mu=\mu_{i}$ and $\Sigma_{i}=\Sigma$ for all $i=1, \ldots, n$, therefore $q_{\alpha, i}$ does not depend to $i$. It will depend only to the $q_{\alpha}$ given by the mixture of elliptic VaR. In effect, $q_{\alpha, i}=q_{\alpha}=q_{\alpha}^{M E-V a R_{\alpha}}$ such that

$$
V a R_{\alpha}=-\delta \cdot \mu+q_{\alpha}^{M E-V a R_{\alpha}} \cdot \sqrt{\delta \Sigma \delta^{t}}
$$

We therefore obtain the following corollary:

Corollary 4.3. Suppose that the portfolio is linear in the risk-factors $X=\left(X_{1}, \cdots, X_{n}\right)$ : $\Delta \Pi=\delta \cdot X$ and that $X \sim N(\mu, \Sigma, \phi)$, with pdf $f_{X}(x)=\sum_{i=1}^{m} \beta_{i}|\Sigma|^{-1 / 2} g_{i}((x-$ $\left.\mu) \Sigma^{-1}(x-\mu)^{t}\right)$. If we replace $q_{\alpha}$ by his value, then the expected Shortfall at level $\alpha$ is given by:

$$
E S_{\alpha}=-\delta \cdot \mu+q_{\alpha}^{M E-E S} \cdot \sqrt{\delta \Sigma \delta^{t}}
$$

where

$$
q_{\alpha}^{M E-E S}=\frac{\pi^{\frac{n-1}{2}}}{\alpha \cdot \Gamma\left(\frac{n+1}{2}\right)} \sum_{i=1}^{m} \beta_{i} \cdot \int_{\left(q_{\alpha}^{M E-V a R}\right)^{2}}^{\infty}\left(u-\left(q_{\alpha}^{M E-V a R}\right)^{2}\right)^{\frac{n-1}{2}} g_{i}(u) d u
$$

4.1. Application: Mixture of Student- $t$ Expected Shortfall. In the case of multi-variate t-student distributions we have that $g_{i}(u)=C\left(\nu_{i}, n\right)\left(1+u / \nu_{i}\right)^{-\frac{\left(n+\nu_{i}\right)}{2}}$, with $C\left(\nu_{i}, n\right)$ given in section 2 . Let us momentarily write $q$ for $q_{\alpha, \nu_{i}}^{\mathfrak{t}}$. Following [3], we can evaluate the integral as follows:

$$
\begin{aligned}
& \int_{q^{2}}^{\infty}(u-q)^{\frac{n-1}{2}}\left(1+\frac{u}{\nu_{i}}\right)^{-\frac{n+\nu_{i}}{2}} d u \\
= & \nu_{i}^{\frac{n+\nu_{i}}{2}}\left(q^{2}+\nu_{i}\right)^{-\left(\frac{\nu_{i}-1}{2}\right)} B\left(\frac{\nu_{i}-1}{2}, \frac{n+1}{2}\right) .
\end{aligned}
$$

If we pose that :

$$
q_{\alpha}^{M S T-E S}=\frac{1}{\alpha \cdot \sqrt{\pi}} \sum_{i=1}^{m} \beta_{i} \frac{\Gamma\left(\frac{\nu_{i}-1}{2}\right)}{\Gamma\left(\frac{\nu_{i}}{2}\right)} \nu_{i}^{\nu_{i} / 2}\left(\left(q_{\alpha, \nu_{i}}^{\mathrm{t}}\right)^{2}+\nu_{i}\right)^{\frac{1-\nu_{i}}{2}}
$$

After substitution in (8), we find, after some computations, the following result:

Theorem 4.4. The Expected Shortfall at confidence level $1-\alpha$ for a multi-variate Student-distributed linear portfolio $\delta \cdot X$, with

$$
f_{X}(x)=\sum_{i=1}^{m} \beta_{i} \frac{\Gamma\left(\frac{\nu_{i}+n}{2}\right)}{\Gamma\left(\nu_{i} / 2\right) \cdot \sqrt{|\Sigma|\left(\nu_{i} \pi\right)^{n}}}\left(1+\frac{(x-\mu)^{t} \Sigma^{-1}(x-\mu)}{\nu_{i}}\right)^{-\left(\frac{\nu_{i}+n}{2}\right)},
$$


is given by:

$$
\begin{aligned}
E S_{\alpha, \nu}^{\mathrm{t}} & =-\delta \cdot \mu \sum_{i=1}^{m} \beta_{i}+\left|\delta \Sigma \delta^{t}\right|^{1 / 2} \sum_{i=1}^{m} \beta_{i} \frac{1}{\alpha \cdot \sqrt{\pi}} \frac{\Gamma\left(\frac{\nu_{i}-1}{2}\right)}{\Gamma\left(\frac{\nu_{i}}{2}\right)} \nu_{i}^{\nu_{i} / 2}\left(\left(q_{\alpha}^{M S T-V a R}\right)^{2}+\nu_{i}\right)^{\left(\frac{1-\nu_{i}}{2}\right)} \\
& =-\delta \cdot \mu+\left|\delta \Sigma \delta^{t}\right|^{1 / 2} \sum_{i=1}^{m} \frac{\beta_{i}}{\alpha \cdot \sqrt{\pi}} \frac{\Gamma\left(\frac{\nu_{i}-1}{2}\right) \nu_{i}^{\nu_{i} / 2}}{\Gamma\left(\frac{\nu_{i}}{2}\right)}\left(\left(\frac{\delta \cdot \mu+V a R_{\alpha}}{|\delta \Sigma \delta|^{1 / 2}}\right)^{2}+\nu_{i}\right)^{\left(\frac{1-\nu_{i}}{2}\right)} \\
& =-\delta \cdot \mu+q_{\alpha}^{M S T-E S} \cdot \sqrt{\delta \Sigma \delta^{t}}
\end{aligned}
$$

The Expected Shortfall for a linear Student portfolio is therefore given by a completely explicit formula, once the $\mathrm{VaR}$ is known. Observe that, as for the VaR, the only dependence on the portfolio dimension is through the portfolio mean $\delta \cdot \mu$ and the portfolio variance $\delta \Sigma \delta^{t}$.

\subsection{Some Numerical Result of Delta Mixture-Student Expected Short-}

fall. Here we give some numerical results when applying the corollary 3.2, in the situation where $m=2$.

For given $s=q_{\beta, \nu_{1}, \nu_{2}}=q_{\alpha}^{M S-V a R}$, which is the solution of

$$
F\left(s, \beta, \nu_{1}, \nu_{2}\right)=\alpha,
$$

by introducing the fonction $\mathrm{H}$ such that

$$
H\left(s, \beta, \nu_{1}, \nu_{2}\right)=\beta \cdot H_{1}(s)+(1-\beta) \cdot H_{2}(s),
$$

where

$$
H_{j}(s)=\frac{\beta_{i}}{\alpha \cdot \sqrt{\pi}} \frac{\Gamma\left(\frac{\nu_{i}-1}{2}\right)}{\Gamma\left(\frac{\nu_{i}}{2}\right)} \nu_{i}^{\nu_{i} / 2}\left(s^{2}+\nu_{i}\right)^{\frac{1-\nu_{i}}{2}}
$$

\begin{tabular}{|c|c|c|c|c|c|}
\hline & $\overline{(2,3)}$ & $\overline{(3,4)}$ & $(4,6)$ & $(7,15)$ & \\
\hline & 6.36587 & 1.29375 & 0.243125 & .00290856 & 0.000681262 \\
\hline & 7.01881 & 4100 & 279 & 0.00341273 & .000 \\
\hline & 7.64714 & 1.52252 & 0.31424 & 0.00389277 & $\overline{0.00}$ \\
\hline & 8.25196 & 63141 & 0.34759 & 0.00 & 0.000997532 \\
\hline$q_{0.4}^{M}$ & 8.83444 & 1.73679 & 0.379538 & 0.00478369 & 0.00108926 \\
\hline & 9.3957 & 83877 & 0.410131 & 9619 & 0.00117468 \\
\hline
\end{tabular}

for $j=1,2$. For given as inputs $\beta, \nu_{1}$ and $\nu_{2}$, we give a table that contains some values of $q_{\alpha}^{M S T-E S}=H\left(q_{\alpha}^{M S T-V a R}, \beta, \nu_{1}, \nu_{2}\right)=q_{\beta, \nu_{1}, \nu_{2}}^{M S T-E S}$.

(1) In the case where $\alpha=0.01$, we obtain some solutions of (4.2) in the following table:

(2) In the case where $\alpha=0.001$, we obtain some solutions of (4.2) in the following table:

\begin{tabular}{|c|c|c|c|c|c|}
\hline$\left(\nu_{1}, \nu_{2}\right)$ & $(2,3)$ & $(3,4)$ & $(4,6)$ & $(7,15)$ & $(8,40)$ \\
\hline$q_{0.25,2,-E S}^{M S T}, \nu_{2}$ & 20.8961 & 3.03289 & 0.576689 & 0.00661826 & 0.00164597 \\
\hline$q_{0.30, \nu_{1}, \nu_{2}}^{M S T-E S}$ & 23.1642 & 3.32289 & 0.666054 & 0.0074621 & 0.00180969 \\
\hline$q_{0.353, \nu_{1}, E S}^{M S T}$ & 25.2707 & 3.58757 & 0.716427 & 0.008196 & 0.00194229 \\
\hline$q_{0.40, \nu_{1}, \nu_{2}}^{M S T}$ & 27.239 & 3.83719 & 0.776394 & 0.00883632 & 0.00205071 \\
\hline$q_{0.45, \nu_{1}, \nu_{2}}^{M S T}$ & 29.0885 & 4.07077 & 0.830853 & 0.00939711 & 0.00214048 \\
\hline$q_{0.50, \nu_{1}, \nu_{2}}^{M S T}$ & 30.8351 & 4.28993 & 0.880508 & 0.00989055 & 0.00221577 \\
\hline
\end{tabular}


4.3. Delta-Theta Approximation of a Portfolio. In the case where we dealt with portfolio that contains derivatives, we will consider the Greek Theta of the portfolio by replace the Delta approximation known in financial literature by the Delta-Theta approximation.

In clear, suppose that we are holding a portfolio of derivatives depending on $n$ underlying assets $X(t)=\left(X_{1}(t), \ldots, X_{n}(t)\right)$, with elliptically distributed logreturns $r_{j}=\log \left(X_{j}(t) / X_{j}(0)\right)$, over some fixed small time-window $[0, \mathrm{t}]$. The portfolio's present value $V$ will in general be some complicated non-linear function of the $X_{i}$ 's. To obtain a first approximation of its VaR, we simply approximate the present Value $\mathrm{V}$ of the position using a first order Taylor expansion:

$$
\begin{aligned}
V(X(t), t) & \approx V(X(0), 0)+\sum_{i=1}^{n} \frac{\partial V}{\partial X_{i}}(X(0), 0)\left(X_{i}(t)-X_{i}(0)\right)+t \cdot \frac{\partial V}{\partial t}(X(0), 0) \\
& =V(X(0), 0)+\sum_{i=1}^{n} \frac{\partial V}{\partial X_{i}}(X(0), 0) X_{i}(0)\left(\exp \left(r_{i}\right)-1\right)+\Theta \cdot t \\
(12) & \approx V(X(0), 0)+\sum_{i=1}^{n} \delta_{i} r_{i}+\Theta \cdot t
\end{aligned}
$$

From this, we can then approximate the Profit \& Loss function as

$$
\Delta V \approx \delta \cdot r^{t}+\Theta \cdot t
$$

where we put $r=\left(r_{1}, \ldots, r_{n}\right)$ and $\delta=\left(\delta_{1}, \ldots, \delta_{n}\right)$ with $\delta_{i}=X_{i}(0) \cdot \frac{\partial V}{\partial X_{i}}(X(0), 0)$. The entries of the $\delta$ vector are called the "delta equivalents "for the position, and they can be interpreted as the sensitivities of the position with respect to changes in each of the risk factors. In this particular case, we have substitute the Delta normal VaR as known in the financial literature, by the Delta-Theta Elliptic VaR given by the following corollary of the theorem (2.1) :

Corollary 4.5. Suppose that the portfolio's Profit \& Loss function over the time window of interest is, to good approximation, given by $\Delta \Pi=\delta \cdot r^{t}+\Theta \cdot t$, with constant portfolio weights $\delta=\left(\delta_{1}, \ldots, \delta_{n}\right)$. Suppose moreover that the random vector $r=\left(r_{1}, \cdots, r_{n}\right)$ of underlying log-returns follows a continuous elliptic distribution, with probability density given by $f_{r}(x)=|\Sigma|^{-1 / 2} g\left((x-\mu) \Sigma^{-1}(x-\mu)^{t}\right)$ where $\mu$ is the vector mean and $\Sigma$ is the variance-covariance matrix, and where we suppose that $g\left(s^{2}\right)$ is integrable over $\mathbb{R}$, continuous and nowhere 0 . Then the portfolio's Delta-Theta-elliptic VaR $\operatorname{VaR}_{\alpha}$ at confidence $1-\alpha$ is given by

$$
\operatorname{VaR}_{\alpha}=-\delta \cdot \mu^{t}+\Theta \cdot t+q_{\alpha, n}^{g} \cdot \sqrt{\delta \Sigma \delta^{t}},
$$

where $s=q_{\alpha, n}^{g}$ is the unique positive solution of the transcendental equation

$$
\alpha=G\left(q_{\alpha, n}^{g}\right) \text {. }
$$

The Expected Shortfall of such portfolios is given by the following corollary

Corollary 4.6. Suppose that the portfolio's Profit \& Loss function over the time window of interest is, to good approximation, given by $\Delta \Pi=\delta \cdot r^{t}+\Theta \cdot t$, and that $r \sim N(\mu, \Sigma, \phi)$, with pdf $f(x)=|\Sigma|^{-1 / 2} g\left((x-\mu) \Sigma^{-1}(x-\mu)^{t}\right)$, then the Delta-Theta Elliptic Expected Shortfall or Delta-Theta ES at confidence level $1-\alpha$ is :

$$
E S_{\alpha}=-\delta \cdot \mu^{t}+\Theta \cdot t+\left|\delta \Sigma \delta^{t}\right|^{1 / 2} \cdot \frac{\pi^{\frac{n-1}{2}}}{\alpha \cdot \Gamma\left(\frac{n+1}{2}\right)} \cdot \int_{\left(q_{\alpha, n}^{g}\right)^{2}}^{\infty}\left(u-\left(q_{\alpha, n}^{g}\right)^{2}\right)^{\frac{n-1}{2}} g(u) d u
$$

Remark 4.7. In short-term Risk Management, one can usually assume that $\mu \simeq 0$. In that case, for $t=1$ we have

$$
\operatorname{VaR}_{\alpha}=\Theta+\sqrt{\delta \Sigma \delta^{t}} \cdot q_{\alpha, n}^{g},
$$




$$
E S_{\alpha}=\Theta+\left|\delta \Sigma \delta^{t}\right|^{1 / 2} \cdot \frac{\pi^{\frac{n-1}{2}}}{\alpha \cdot \Gamma\left(\frac{n+1}{2}\right)} \cdot \int_{\left(q_{\alpha, n}^{g}\right)^{2}}^{\infty}\left(u-\left(q_{\alpha, n}^{g}\right)^{2}\right)^{\frac{n-1}{2}} g(u) d u .
$$

As before, The preceding can immediately be specialized to a Student $\mathfrak{t}$-distributions to estimate the Delta-Theta Student VaR and the Delta-Theta Student ES. The details will be left to the reader.

4.4. Portfolios of Equities. A special case of the preceding is that of an equity portfolio, build of stock $S_{1}, \ldots, S_{n}$ with joint log-returns $r=\left(r_{1}(t), \ldots, r_{n}(t)\right)$. In this case, the portfolio's Profit \& Loss function over the time window $[0, t]$ of interest is, to good approximation, given by

$$
\begin{aligned}
\Pi(t)-\Pi(0) & =\sum_{i=1}^{n} w_{i} S_{i}(0)\left(S_{i}(t) / S_{i}(0)-1\right) \\
& \approx \sum_{i=1}^{n} w_{i} S_{i}(0) r_{i}(t)=\delta \cdot r^{t}
\end{aligned}
$$

where this approximation will be good if the $r_{i}(t)$ are small. In this case the preceded theorems are applicable where $\delta=\left(w_{1} S_{1}(0), \ldots, w_{n} S_{n}(0)\right)$ and $r_{j}(t)=$ $\log \left(X_{j}(t) / X_{j}(0)\right)$ for $\mathrm{j}=1, \ldots, \mathrm{n}$.

4.5. Businesses as Linear Portfolios of Business Units. An interesting way of looking upon an big enterprize, e.g. a multi-national or a big financial institution, is by considering it as a sum of its individual business units, cf. Dowd [2]. If $X_{j}$, is the variation of price or of profitability of business unit $j$ in one period, then the variation of price of the agglomerate in the same period will be

$$
\Delta \Pi=X_{1}+\cdots+X_{n}
$$

The entire institution is therefore modelled by a linear portfolio, with $\delta=(1,1, \ldots, 1)$, to which the results of this paper can be applied, if we model the vector of individual price variations by a multi-variate elliptic distribution. VaR, incremental VaR (see below) and Expected Shortfall will be relevant here. For more details see Dowd [2], chapter XI .

4.6. Incremental VaR. Incremental VaR is defined in [1] as the statistic that provides information regarding the sensitivity of $\mathrm{VaR}$ to changes in the portfolio holdings. It therefore gives an estimation of the change in VaR resulting from a risk management decision. Results from [1] for incremental VaR with normally distributed risk-factors generalize straightforwardly to elliptically distributed ones: if we denote by $I V a R_{i}$ the incremental VaR for each position in the portfolio, with $\theta_{i}$ the percentage change in size of each position, then the change in VaR will be given by

$$
\Delta V a R=\sum \theta_{i} I V a R_{i}
$$

By using the definition of $I V a R_{i}$ as in [1] (2001), we have that

$$
I V a R_{i}=\omega_{i} \frac{\partial V a R}{\partial \omega_{i}}
$$

with $\omega_{i}$ is the amount of money invested in instrument $i$. In the case of an equity portfolio in the elliptically distributed assets, we have seen that, assuming $\mu=0$,

$$
\operatorname{VaR}_{\alpha}=-q_{\alpha, n}^{g} \sqrt{\delta \Sigma \delta^{t}}
$$

We can then calculate $I V a R_{i}$ for the i-th constituent of portfolio as

$$
I V a R_{i}=\omega_{i} \frac{\partial V a R}{\partial \omega_{i}}=\omega_{i} \gamma_{i}
$$


with

$$
\gamma=-q_{\alpha, n}^{g} \frac{\Sigma \omega}{\sqrt{\delta \Sigma \delta^{t}}} .
$$

The vector $\gamma$ can be interpreted as a gradient of sensitivities of VaR with respect to the risk factors. This is the same as in [1], except of course that the quantile has changed from the normal one to the one associated to $g$.

4.7. Problem of the aggregation of risks. Suppose that we have a constituted portfolio with several under portfolios of assets from different markets. Given the Value-at-Risk of the portfolios constituting the global portfolio, under the hypothesis that the joined risks factors follow an elliptic distribution, the question is how to get the VaR of the global portfolio.

In order to be clearer and simpler, let us consider a global constituted portfolio of 2 under portfolios from different markets with respective weights $\delta_{\mathbf{1}}$ and $\delta_{\mathbf{2}} . \boldsymbol{\Sigma}_{\mathbf{1}}$ represents the matrix of interrelationship in the under portfolio of market $1 ; \boldsymbol{\Sigma}_{\mathbf{2}}$ represents the matrix of interrelationship in the under portfolio of market 2 . One will be able to write the matrix of interrelationship of a global portfolio like this:

$$
\Sigma=\left(\begin{array}{cc}
\Sigma_{1} & \Sigma_{12} \\
\Sigma_{12} & \Sigma_{2}
\end{array}\right)
$$

where $\boldsymbol{\Sigma}_{\mathbf{1 2}}$ is the correlation matrix that takes into consideration the interaction between the market $\mathbf{M}_{\mathbf{1}}$ and the market $\mathbf{M}_{\mathbf{2}}$. If $\delta^{t}=\left(\delta_{\mathbf{1}}, \delta_{\mathbf{2}}\right)$, we have

$$
\delta^{t} \boldsymbol{\Sigma} \delta=\delta_{\mathbf{1}}{ }^{t} \boldsymbol{\Sigma}_{\mathbf{1}} \delta_{\mathbf{1}}+\delta_{\mathbf{2}}{ }^{t} \boldsymbol{\Sigma}_{\mathbf{2}} \delta_{\mathbf{2}}+2 \cdot \delta_{\mathbf{1}}{ }^{t} \boldsymbol{\Sigma}_{\mathbf{1 2}} \delta_{\mathbf{2}} .
$$

Therefore, since we know that when $\mu \approx 0$, we have

$$
V a R_{\alpha}=q_{\alpha, n}^{g} \cdot \sqrt{\delta \Sigma \delta^{t}}
$$

the Value-at-Risk of the global portfolio will be given by

$$
\operatorname{VaR}_{\alpha}(\mathbf{M})=\sqrt{\operatorname{VaR}_{\alpha}\left(\mathbf{M}_{\mathbf{1}}\right)^{2}+\operatorname{VaR}_{\alpha}\left(\mathbf{M}_{\mathbf{2}}\right)^{2}+\mathbf{2}\left[\mathbf{q}_{\alpha, \mathbf{n}}^{\mathrm{g}}\right]^{\mathbf{2}} \cdot \delta_{\mathbf{1}}{ }^{\mathrm{t}} \boldsymbol{\Sigma}_{\mathbf{1 2}} \delta_{\mathbf{2}}}
$$

An implicit interrelationship with the hypothesis of elliptic distribution is obtained in an analogous way, like in the case where one works with the hypothesis of the normal distribution. Note that, one will distinguish several situations from the behavior of $\boldsymbol{\Sigma}_{\mathbf{1 2}}$. With some simple operations, the implicit interrelationship is

$$
\phi=\frac{\delta_{\mathbf{1}}{ }^{t} \boldsymbol{\Sigma}_{\mathbf{1 2}} \delta_{\mathbf{2}}}{\sqrt{\left(\delta_{\mathbf{1}}{ }^{t} \boldsymbol{\Sigma}_{\mathbf{1}} \delta_{\mathbf{1}}\right)\left(\delta_{\mathbf{2}}{ }^{t} \boldsymbol{\Sigma}_{\mathbf{2}} \delta_{\mathbf{2}}\right)}}
$$

with the Value-at-Risk $\operatorname{VaR}_{\alpha}(\mathbf{M})$ of the global portfolio being given as follows:

$$
\operatorname{VaR}_{\alpha}(\mathbf{M})=\sqrt{\left.\left[\operatorname{VaR}_{\alpha}\left(\mathbf{M}_{\mathbf{1}}\right)\right]^{2}+\left[\operatorname{VaR}_{\alpha}\left(\mathbf{M}_{\mathbf{2}}\right)\right]^{2}+\mathbf{2} \phi \cdot \operatorname{VaR}_{\alpha}\left(\mathbf{M}_{\mathbf{1}}\right) \operatorname{VaR}_{\alpha}\left(\mathbf{M}_{\mathbf{2}}\right)\right)} .
$$

Also, for $\mu \approx 0$,

$$
E S_{\alpha}=K_{E S, \alpha}^{g} \cdot \sqrt{\delta \Sigma \delta^{t}}
$$

therefore by using the same technics that proves (16), we have that the expected shortfall of the global portfolio is given by:

$$
E S_{\alpha}(\mathbf{M})=\sqrt{\mathbf{E S}_{\alpha}\left(\mathbf{M}_{1}\right)^{2}+\mathbf{E S}_{\alpha}\left(\mathbf{M}_{2}\right)^{2}+\mathbf{2}\left[\mathbf{K}_{\mathbf{E S}, \alpha}^{\mathrm{g}}\right]^{2} \cdot \delta_{\mathbf{1}}{ }^{\mathrm{t}} \boldsymbol{\Sigma}_{12} \delta_{2}} .
$$

This imply that

$$
E S_{\alpha}(\mathbf{M})=\sqrt{\left.\left[\mathbf{E S}_{\alpha}\left(\mathbf{M}_{\mathbf{1}}\right)\right]^{\mathbf{2}}+\left[\mathbf{E S}_{\alpha}\left(\mathbf{M}_{\mathbf{2}}\right)\right]^{\mathbf{2}}+\mathbf{2} \phi_{\mathbf{E S}} \cdot \mathbf{E S}_{\alpha}\left(\mathbf{M}_{\mathbf{1}}\right) \mathbf{E S}_{\alpha}\left(\mathbf{M}_{\mathbf{2}}\right)\right)}
$$


where

$$
\phi_{E S}=\frac{\delta_{\mathbf{1}}{ }^{t} \boldsymbol{\Sigma}_{\mathbf{1 2}} \delta_{\mathbf{2}}}{\sqrt{\left(\delta_{\mathbf{1}}{ }^{t} \boldsymbol{\Sigma}_{\mathbf{1}} \delta_{\mathbf{1}}\right)\left(\delta_{\mathbf{2}}{ }^{t} \boldsymbol{\Sigma}_{\mathbf{2}} \delta_{\mathbf{2}}\right)}}
$$

Remark 4.8. The result about the agregation of risks work so well in the situation where, the joint risk factors of our portfolio changes with mixture of ellitic distributions as define in (2.3), and where all $\Sigma_{i}=\Sigma$, for $i=1, \ldots, m$. In particular, when $\mu_{i}=\mu$, we have the results (20) and (16).

\section{CONCLUSION}

In this paper, we have shown how to reduce the estimation of Value-at-Risk for linear elliptic portfolios to the evaluation of one dimensional integrals which, for the special case of a mixture of $t$-Student distributions, can be explicitly evaluated in terms of a hypergeometric function. We have also given a similar, but simpler, integral formula for the expected shortfall of such portfolios which, again, can be completely evaluated in the Student case. Following the calculations in the case of Delta mixture-Student VaR, we indicated how to extend it to the case of mixture of t-distributions expected shortfall . We finally surveyed some potential application areas. 


\section{REFERENCES}

1] J.Mina and J.Y.Xiao (2001), Return to Riskmetrics: The Evolution of the standard (www.riskmetrics.com). 2001 RiskMetrics Group Inc.

[2] K.Dowd,(1998) Beyond Value-at-Risk: the new science of risk Management (Wiley series in Frontiers in Finance).

[3] J.Sadefo-Kamdem, (2003) Value-at-Risk and Expected Shortfall for Linear Portfolio with Elliptically Distributed Risk Factors,submitted

[4] J.Sadefo-Kamdem, (2003): Value-at-Risk and Expected Shortfall for quadratic portfolio of securities with mixture of elliptic distributed risk factors. Submitted.

[5] Subu Venkataraman, (1998): Value at risk for a mixture of normal distributions: The use of quasi-bayesian estimation techniques. Economic Perspectives

[6] Yasuhiro and Toshinao Yoshiba, (2002): On the validity of Value-at-Risk: Comparative analysis with Expected shortfall

7] Zangari, Peter, " An Improved methodology for measuring VaR," RiskMetrics Monitor, 2nd quarter pp.7-25. http://www.riskmetrics.com/research/journals 